\title{
共情：遗传-环境-内分泌-大脑机制
}

杨业 ${ }^{1,2,3}$, 汤艺 ${ }^{1}$, 彭微微 ${ }^{4}$, 吕雪靖 ${ }^{2,3^{*}}$, 胡理 ${ }^{1,2,3^{*}}$, 陈军 ${ }^{5}$

1. 西南大学心理学部, 认知与人格教育部重点实验室, 重庆 400715;

2. 中国科学院心理健康重点实验室(中国科学院心理研究所), 北京 100101;

3. 中国科学院大学心理学系, 北京 100049;

4. 深圳大学心理与社会学院, 脑功能与心理科学研究中心, 深圳 518060;

5. 第四军医大学唐都医院疼痛生物医学研究所, 西安 710038

* 联系人, E-mail: luxj@psych.ac.cn; huli@psych.ac.cn

2017-08-01 收稿, 2017-09-03 修回, 2017-09-04 接受, 2017-10-13 网络版发表

国家自然科学基金(31471082,3167114)、重庆市基础与前沿研究计划(cstc2015jcyjBX0050)、中国科学院心理研究所科研启动项目(Y6CX021008) 和中国科学院心理研究所青年人才科研启动经费(Y6CX281007)资助

摘要 共情是指感知到他人的情感状态, 使得自己产生与之类似的情绪或感受的能力, 它包含情感共情和认知 共情两种成分. 本文结合健康被试和脑损伤患者的神经影像学研究, 概述了共情两种成分的认知神经基础: 情感 共情涉及的特异性脑区主要有额下回(IFG)、脑岛和前扣带回(ACC), 认知共情的特异性脑区主要有腹内侧前额叶 皮层(vmPFC). 然后, 从内分泌、遗传和环境几个方面, 详细讨论了影响共情能力及其行为的复杂因素. 对共情的 内分泌研究发现，催产素能够促进个体的共情反应，而睪丸素则会弱化个体的共情反应，且产前睪丸素可能会影 响个体共情能力的发育; 对共情的社会环境和遗传学研究发现, 家庭成员的特质、父母教养方式、学校教育和人 际关系等环境因素与催产素受体、多巴胺受体、五羟色胺转运蛋白(5-HTTLPR)和锌指蛋白(ZNF804A)等基因的多 态性都会影响共情反应和共情能力的发展. 在现有研究的基础上, 本文提出了共情的遗传-环境-内分泌-大脑理论 框架, 揭示了影响共情的各个因素间的相互作用. 最后, 指出了当前共情研究中所存在的问题和局限, 未来有必 要在大样本研究的基础上开展纵向研究, 综合考量多个因素(遗传、环境、内分泌和大脑)及其交互作用对共情的 影响, 揭示共情能力提升的关键因素; 并在此基础上促进基础共情研究成果向社会生活和临床实践转化, 有效地 改善个体的共情能力及其行为, 从而促进社会和谐与进步, 真正意义上实现“老吾老, 以及人之老; 幼吾幼, 以及 人之幼”。

关键词 共情, 情感共情, 认知共情, 大脑, 环境, 基因

作为人类情绪体验和社会交往中的一个关键成 分, 共情能让我们分享他人的情绪状态, 推测和理解 他人的情绪、动机和行为 ${ }^{[1]}$. 目前, 共情被广泛定义 为: 感知到他人的情感状态, 从而使得自己产生与之 类似的情绪或感受的能力 ${ }^{[2]}$. 据此, 共情可以分为两 种不同的成分, 即情感共情和认知共情. 情感共情 (affective empathy)是指个体对他人情绪的识别和判
断, 包括情绪感染和情感观点采择; 认知共情 (cognitive empathy)则倾向于设身处地地理解他人的 情感, 并区分这种情感是源于自己还是他人，与情感 心理理论(theory of mind, ToM) 类似 ${ }^{[3]}$. 情感共情是 一种较原始初级的共情, 婴儿出生时便具备这种能 力，其发展依赖于镜像神经元系统 (mirror neuron system)中共享表征的日益丰富和精细. 相比较之下, 
认知共情是一种更为高级的共情, 在儿童或青少年 期不断发展, 老年期呈逐渐下降的趋势, 随着颞极联 合区和前额叶皮层的成熟, 以及个体区分自我-他人 和抑制自我中心能力的增强而逐渐发展 ${ }^{[4]}$. 共情的两 种成分相互补充、协调工作, 其中任何一种受损或者 缺失都会导致个体在情感方面的障碍或失调. 例如 孤独症 (autism spectrum disorders, ASD)患者和精神 分裂症患者都会表现出不同程度的情感障碍和失调. 前者的情感共情正常, 但是认知共情缺失, 表现为难 以推测他人的心理状态和理解他人的观点 ${ }^{[5]}$; 而后 者的认知共情完好, 但是情感共情缺失, 主要表现为 难以识别他人的情绪 ${ }^{[6]}$. 此外, 也有研究将共情分为 特质共情和状态共情 ${ }^{[7,8]}$. 其中, 特质共情指个体所 拥有的比较稳定的心理特性, 是个体对他人的情绪 和情感状态产生共鸣的一种能力; 而状态共情是指 一种由相应的情景所引发的心理状态, 主要强调个 体与环境交互过程中所产生的共情反应.

由于共情是激发亲社会行为和抑制攻击行为的 关键, 为个体道德发展提供了情感和动机基础 ${ }^{[9]}$, 对 维持社会和谐起着重要的作用. 近年来, 共情受到心 理学、脑神经科学、分子生物学、社会环境学和遗传 学等领域的广泛关注 ${ }^{[10]}$, 并在相关领域取得了突破 性进展. 本文将共情看作一种能力 (包含特定情境下 的共情反应), 从情感共情和认知共情两个方面出发, 首先对共情的神经影像研究进行归纳和总结, 然后 重点综述了近年来在共情的神经生物学、社会环境学 和遗传学方面所取得的进展, 最后提出了共情的遗 传-环境-内分泌-大脑理论框架, 并指出了当前共情 研究存在的问题和局限, 也为未来的研究方向和方 法提出了意见和建议.

\section{1 情感共情和认知共情的大脑机制}

情感共情和认知共情有着共同的脑网络基础, 但这两种成分涉及不同的脑区. 通过分析对比健康 个体在特定共情任务中各个脑功能区的激活程度, 研究发现, 情感共情主要涉及负责情绪状态匹配和 情感共享的脑区, 如脑岛(insula)、前扣带回(anterior cingulate cortex, ACC)、杏仁核(amydala)和额下回 (inferior frontal gyrus, IFG $)^{[11 \sim 14]}$; 认知共情主要涉及 的脑区则更多参与精细、可控的认知加工, 如腹内侧 前额叶(ventromedial prefrontal cortex, vmPFC) ${ }^{[15]}$. 然 而, 在某种特定共情任务下所激活的脑区并不意味
着该脑区是共情的特异性脑区, 因为其无法提供直 接证据证明该脑区的损伤或者缺失会影响共情能 力 $^{[16]}$. 在这种情况下, 相关脑区损伤或脑结构异常 的案例为共情特异性脑区的研究提供了新的思路和 方向 ${ }^{[17,18]}$. 通过分析特定脑区的结构或功能的缺失 或异常如何影响共情能力, 可以有效鉴别参与共情 的特异性脑区. 因此, 本部分将结合大脑损伤或异常 患者和健康被试的神经影像研究结果, 讨论共情的 认知神经基础.

\section{1 情感共情}

情感共情包含情绪感染和情感观点采择两个方 面, 前者通常是指个体对自我情感状态的抑制以及 对他人情感状态的识别, 但这种识别并不一定能被 个体意识到; 而后者是指抑制个体的自我观点, 通过 观察或想象来识别他人的情感状态 ${ }^{[19]}$.

\subsection{1 额下回(IFG)}

IFG 是额叶脑回的一部分, 主要位于布鲁德曼 44, 45和47区(BA44, 45, 47), 涉及语义的加工和风险 规避等认知过程 ${ }^{[20,21]}$. 此外, 有研究显示 IFG的激活 与情感共情相关, 尤其体现在其右侧区域对情绪的 识别和感染方面 ${ }^{[22]}$, 这可能是由于这个区域中的镜 像神经元对识别面部表情和模仿他人至关重要, 而 这种模仿正是发展情绪感染的基础 ${ }^{[23]}$. 共情者会根 据其与被共情者的熟悉性、共同特征或经历、性别以 及潜在的重要性等来调节自身的共情水平, 并表现 为不同程度的 IFG激活 ${ }^{[24]}$. 例如, Chakrabarti等人 ${ }^{[25]}$ 发现被试在共情量表中的得分越高(即共情能力越 强), 在观看动态情绪视频(如快乐、悲伤、生气和厌 恶等)时IFG的激活程度越强. 类似地, Jabbi等人 ${ }^{[26]}$ 发 现, 被试在观看静态积极和厌恶表情时, IFG激活, 且激活程度可以由被试在共情量表上得分的高低预 测. 这些研究都表明了 IFG参与情感共情任务的加 工. Shamay-Tsoory等人 ${ }^{[14]}$ 对 IFG病变患者和前额叶 病变患者的研究, 进一步证明了 IFG的激活对共情的 必要性. 该研究中, 相对于健康被试, IFG病变患者 在情绪感染和识别方面受到了损害, 表现为人际反 应指针量表(interpersonal reactivity index, IRI)中情绪 共情分量表部分得分较低, 且在共情相关任务中无 法正确识别他人的情绪表情. 更重要的是, 非IFG病 变的前额叶病变患者在IRI情绪共情分量表部分的得 分和表情识别任务中与正常被试的表现并无显著差 
异, 表明IFG是情感共情的特异性脑区.

\subsection{2 脑岛和前扣带回(ACC)}

脑岛是向内凹陷的皮层区域，位于外侧沟深面， 主要涉及到感觉、意识和决策等过程 ${ }^{[27 ~ 29]}$; ACC 位于 扣带皮质的前部, 包含BA24, 32和33区, 涉及情绪和 认知功能 ${ }^{[30]}$. 近年来很多功能成像的证据表明, 脑 岛和ACC 都会参与对他人情绪的感知 ${ }^{[26,31]}$. 例如, 通 过与眶额叶、前额叶、ACC、颞极和杏仁核的广泛连 接, 脑岛可以对情绪进行整合, 其中右侧前脑岛 (anterior insula, AI) 在调节感觉意识或厌恶感觉方面 有重要作用 ${ }^{[32]}$, 而 ACC不仅与脑岛有紧密的联结, 与眶额叶和杏仁核之间的功能联系也很紧密 ${ }^{[33 \sim 35]}$. 近年来对共情的大脑成像研究发现, 脑岛和 ACC 在 情绪感染和情感观点采择任务中都会被显著激活 ${ }^{[36]}$. 因此脑岛和 $\mathrm{ACC}$ 被认为是参与共情过程的重要脑区. 为了探究脑岛和 $\mathrm{ACC}$ 与共情之间的联系, Kim等人 ${ }^{[37]}$ 通过对额颞叶型失智症 (frontotemporal dementia, FTD)(一种神经退行性疾病, 主要表现为共情能力受 损) 患者的脑岛和 ACC 脑区中的纺锤体神经元 (von economo neurons)进行选择性追踪研究发现, 右侧脑 岛和 $\mathrm{ACC}$ 的纺锤体神经元丧失量与FTD的临床严重 程度显著相关, 证明纺锤体神经元对人类进化出社 会性行为和情绪起着重要作用 ${ }^{[38]}$. Boucher等人 ${ }^{[39]}$ 对 15名因癫㾁而移除脑岛的患者的研究发现, 这些患 者在观看静态情绪面孔图片时, 无法正确识别面孔 图片所表达的情绪, 说明脑岛的移除影响了这些患 者的情绪识别能力. 类似的结果来自于Driscoll等 人 ${ }^{[40]}$ 的研究, 他们采用基于体素的病变症状映射 (voxel-based lesion-symptom mapping)的方法研究了 192名患有局灶性穿透颖脑损伤多年的退休老兵, 结 果发现, 患者在自我报告式情感共情量表中的得分 与其前脑岛的病变体积呈显著负相关. 此外, Leigh 等人 ${ }^{[3]}$ 对 27 名中风患者中风后 $48 \mathrm{~h}$ 之内的研究发现, ACC 病变患者的情感观点采择能力受到了损害, 表 现为这些患者在观看视频或阅读简短故事后, 不能 准确地根据情景去推断他人的情绪状态. 以上研究 结果证明了脑岛和 $\mathrm{ACC}$ 在情绪感染和情感观点采择 中的必要性.

\section{2 认知共情}

认知共情是指理解他人的情感, 并推断他人可 能的心理或情感状态的一种能力 ${ }^{[15,16]}$. 认知共情主
要涉及心理理论 $(T o M)$, 即指站在他人的视角, 且在 此基础上想象并理解他人的思想和情感的能力 ${ }^{[41]}$. 特别地, 认知共情等同于情感ToM, 即个体对他人情 感的理解 ${ }^{[15]}$. 研究发现, 认知共情会激活 ToM相关 脑区, 如 vmPFC、颞上沟 (superior temporal sulcus, STS)、颢顶交界处(temporoparietal junction, TPJ)、颢 极 (temporal poles, TP)等, 其中 vmPFC对认知共情的 产生尤为重要 ${ }^{[42,43]}$.

vmPFC位于BA10,11，12和25等区域. 研究表明, $\mathrm{vmPFC}$ 参与多种高级认知活动, 例如决策和情绪管 理等 ${ }^{[44,45]}$. 在共情研究中发现, vmPFC对共情过程的 加工非常重要，尤其是对共情的认知成分的加

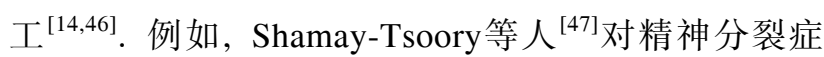
患者的研究发现, 精神分裂症患者的情感和社会行 为方面都受到了损害, 表现为较健康被试在完成情 感 ToM相关任务时的错误率更高, 即 ToM能力的缺 失或错误地理解环境状况. Shamay-Tsoory等人 ${ }^{[48]}$ 随 后又对 24 名患有精神分裂症的患者与 vmPFC病变、背 外侧前额叶皮层病变患者以及健康被试三类人进行 比较, 结果发现, 与健康被试和背外侧前额叶皮层病 变患者相比, 精神分裂症患者和 vmPFC发生病变的 患者在情感ToM任务中出现了相似的错误, 而在认 知 ToM相关的任务当中都表现正常. 为了进一步探 索精神分裂症患者 ToM能力的受损是否与 $\mathrm{vmPFC}$ 异 常相关, Hooker等人 ${ }^{[49]}$ 采用基于体素的形态学测量 (voxel-based morphometry) 和行为评估等方法发现， 相较于健康被试, 患有精神分裂症的被试的 vmPFC 灰质体积更少, 且其在 ToM任务当中的行为表现和 vmPFC灰质体积相关，即患者的 vmPFC灰质体积越 少, 任务的错误率越高.上述研究说明, 精神分裂症 患者大脑 $v m P F C$ 的异常和认知共情能力的损伤非常 相关.

另外, Shamay-Tsoory等人 ${ }^{[43]}$ 对 49 名不同类型大 脑病变患者的共情能力进行研究, 实验中, 要求患者 通过观看一个名叫“Yoni”的卡通人物的眼神和面部 表情去判断“Yoni”在想什么(认知ToM), 或者“Yoni” 对自己周围的人和事物所持有的情感(情感ToM). 结 果发现, 与健康被试相比, vmPFC病变的患者不能准 确地判断“Yoni” 对自己周围的人和事物所持有的情 感, 但是可以正确判断“Yoni”在想什么, 即仅情感 $\mathrm{ToM}$ (认知共情)受到损害. 使用共情问卷对 30 名大脑 发生病变的患者进行研究, 结果与之前研究一致, 发 
现vmPFC病变的患者比vmPFC未发生病变的患者在 共情量表的认知共情部分得分更低, 即 vmPFC患者 认知共情能力受损, 证明了 $\mathrm{vmPFC}$ 在认知共情过程 中的关键作用 ${ }^{[14]}$.

\section{2 共情的内分泌研究}

\section{1 催产素}

催产素(oxytocin)是一种在哺乳动物体内分泌的 激素, 由下丘脑的视上核和室旁核中的大细胞神经 元合成, 在哺乳类动物的社会生活当中起着重要的 作用 ${ }^{[50]}$. 研究发现, 催产素对社会交往、社会记忆、 依恋等具有调节作用 ${ }^{[51 ~ 53]}$. 对健康被试的研究指出, 催产素可以调节情绪识别、压力和社会信任等加工过 程 ${ }^{[50,54]}$; 对临床患者的研究表明, 催产素系统的功能 受到损害将会引起与社会相关的心理障碍, 包括 ASD、社会焦虑障碍、边缘人格障碍和精神分裂症 等 ${ }^{[54]}$. 近年来的研究发现催产素可以调节共情, 从 而促进个体的亲社会行为.

催产素有助于个体对特定情绪的识别. Di Simplicio等人 ${ }^{[55]}$ 和Marsh等人 ${ }^{[56]}$ 的研究均发现, 经鼻给 催产素增强了被试对积极面孔的加工, 但是对负性 面孔的加工并未得到改善. 然而, 当面孔图片在非常 短的时间内呈现时(17 53 ms), 被试不仅对积极(快 乐) 面孔, 对负性 (生气) 面孔的识别准确性也会增 加 ${ }^{[57]}$. 类似地, Fischer-Shofty等人 ${ }^{[58]}$ 发现, 与安慰剂 组相比, 经鼻给催产素增强了被试对恐惧面孔进行 识别的准确性. 尽管目前对催产素是否改善被试对 特定情绪的识别仍存在争议, 但催产素对视觉注意 的影响被认为是情绪识别的关键 ${ }^{[59]}$. 与对照组相比 较, 使用催产素的实验组注视他人眼部的时间更久, 这可能是在经催产素处理后对眼部的注视时间增加 所导致的 ${ }^{[60,61]}$. 然而, 这一研究结果并没有得到重 复 ${ }^{[62,63]}$. 这些研究所得到的矛盾结果可能是由催产 素和个体差异之间相互作用导致的, 即只在特定的 条件下才能调节个体的眼部注视时间, 从而表现为 个体对特定情绪的识别增强.

催产素可以增强杏仁核活动, 从而提高情感共 情. 当被试被要求观看不同的情景图片 (一个带有明 显情绪的人处于一定的情景中), 并根据相应的图片 情景推测图中人的心理状态 (认知共情) 和情绪强度 (情感共情)时, 相比于未使用催产素处理的被试, 鼻
内给催产素的被试在情感共情任务中表现更好, 即 催产素组被试能够更准确地评价他人的情绪强度, 但是在认知共情任务中两组的表现没有显著差异 ${ }^{[13]}$. 然而, 该研究中对杏仁核受到损伤的患者进行催产 素处理后, 被试在情感共情中的表现并未改善, 该 结果表明催产素对情感共情的调节依赖于杏仁核的 加工.

另外, De Dreu等人 ${ }^{[64]}$ 在催产素对团体关系影响 的研究中也取得了很多有意义的发现. 例如, 他们发 现催产素可以通过增强团体内成员间的共情反应、合 作性和一致性，从而调节团体关系. 进一步的研究发 现, 催产素影响团体关系的作用效果主要取决于团 体内成员的共情特质水平 ${ }^{[65]}$, 主要表现为: 与安慰 剂组相比, 经鼻给催产素后, 共情特质较高的团体成 员在做任务决策时会更多地考虑集体利益而更少地 考虑个人利益, 共情特质较低的团体成员则与此相 反, 他们在决策时考虑个人利益较多, 集体利益更 少. 此外, 催产素可以使团体内的成员更加愿意共享 一些独特的信息和见解，从而提高团体决策的速度 和质量 ${ }^{[66]}$.

催产素不仅能够促进健康被试的共情反应, 还 可以增强ASD患者的社会互动能力和对虚拟同伴(虚 拟球游戏当中的同伴)的信任感, 有助于增加患者对 面部图片的眼部注视时间 ${ }^{[67]}$. 给 ASD患者静脉注射 催产素, 可以改善患者对情绪性言语的理解 ${ }^{[68]}$. 当 要求成年ASD患者观看一张只有眼部的图片, 并根据 图片中人物的眼神来判断他们的个人情绪和心理状 态时, 即眼神读心测验(reading the mind in the eyes

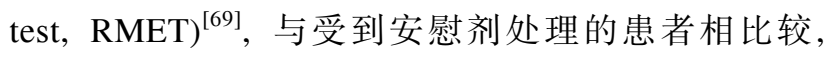
受到催产素处理的患者在RMET当中做出的判断更 加精准, 这种优势在比较有难度的项目中尤其明

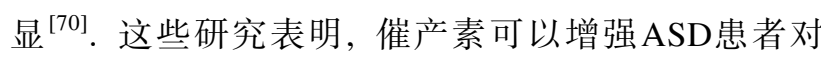
情感的识别、改善对他人情绪状态的反应, 对他们的 主要缺陷可能起到一定的治疗作用.

\section{2 睪丸素}

睪丸素(testosterone)是一种合成类固醇, 主要由 男性睪丸分泌, 女性卵巢或肾上腺也会分泌少量 ${ }^{[71]}$. 据统计，成年男性睪丸素分泌量大约是成年女性分 泌量的20倍, 体内含量是成年女性的7 8倍. 睪丸素 是主要的男性性激素, 对促进男性第二性征的发育 起着关键作用. 研究发现, 睪丸素水平会影响个体的 
共情能力, 男性因为睪丸素水平较高, 其平均共情水 平低于女性, 且此差异从个体出生就存在 ${ }^{[72]}$. 例如, 在观看他人处于痛苦状态时, 一岁的女孩比同龄男 孩表现出更多的悲伤表情和安慰行为 ${ }^{[73]}$. 使用EQ-C (empathy quotient-child version)量表测量发现, 6 8岁 的女孩比男孩的共情能力强 ${ }^{[74]}$.

胎儿在母体中羊水的睪丸素水平, 即产前睪丸 素(fetal testosterone)水平, 影响个体大脑的结构与功 能的发展, 从而影响个体的认知和行为, 例如个体的 系统化程度(systemizing)(分析、理解、预测、控制和 构建系统规则的能力) 和共情能力等 ${ }^{[75]}$. 个体的产前 睪丸素水平越高, 出生后的系统化程度越高, 共情能 力越低 ${ }^{[76,77]}$. Chapman等人 ${ }^{[74]}$ 使用EQ-C量表和RMET 对6 8岁的儿童测量发现, 男孩的产前睪丸素水平与 EQ-C量表和RMET的分数呈显著的负相关, 即产前 睪丸素水平越高的男孩, 其共情能力越弱. 该研究者 认为可能是由于产前睪丸素影响胎儿大脑的结构和 功能的发展, 产前睪丸素水平越高则大脑的系统化 水平越高, 从而导致其共情能力更低. 另一项关于产 前睪丸素的研究主要针对多囊卵巢综合征(polycystic ovary syndrome)患者的女儿进行的. 多囊卵巢综合 征是女性中常见的内分泌紊乱, 主要特点是排卵期 紊乱, 许多小囊肿围绕在卵巢周围(多囊卵巢), 伴随 着雄性激素分泌过多. 对多囊卵巢综合征的女性从 她们怀孕到胎儿出生, 进行为期 5 年的纵向控制研究 发现, 胎儿在子宫内的雄性激素水平较高, 使得胎儿 在出生后有更多的男性化行为, 且在生活当中共情 反应更弱, 这些女孩在共情相关任务中的得分比正 常女性生产的女儿更低 ${ }^{[78]}$. 另两项使用RMET对儿 童的研究一致发现, 无论是男孩还是女孩, 产前睪丸 素的水平越高, 儿童在RMET中得分越低, 即产前睪 丸素水平与共情能力之间存在负相关关系 ${ }^{[74,79]}$.

舌下给睪丸素的研究显示, 睪丸素减弱了成年 女性的 ToM和面部情绪识别能力. 在RMET中, 被试 通过观看图片中人物的眼部区域判断其情绪和心理 状态, 结果显示, 与安慰剂组相比较, 舌下给睪丸素 组在RMET中得分更低, 其共情能力被明显削弱 ${ }^{[80]}$. 也有研究发现, 睪丸素减少了个体对面部表情的模 仿(这被认为是测量情感共情的一个指标) ${ }^{[81]}$, 降低了 个体正确识别他人复杂情绪和情感的准确性 ${ }^{[80]}$. Bos 等人 ${ }^{[82]}$ 使用 $\mathrm{fMRI}$ 研究发现, 与安慰剂组相比较, 睪 丸素的处理弱化了在RMET过程中 IFG与双侧 ACC 和
辅助运动区 (supplementary motor area, SMA)的功能 连接, 该研究者认为IFG-ACC-SMA网络是情绪识别 过程中选择和整合感觉信息的神经基础. 另一项 fMRI的研究中, 被试经过睪丸素处理后, 观看愤怒 表情时, OFC, 杏仁核和下丘脑三个脑区出现了持续 性激活，观看快乐表情时则没有出现这种现象，这表 明了睪丸素可能在社会威胁当中起着较为重要的调 节作用 ${ }^{[83]}$.

\section{3 共情的遗传-环境基础}

目前为止, 大多数研究主要通过相关脑区的激 活程度作为证据来判断个体共情特质及其相关的认 知神经机制, 而对人类共情的遗传和环境基础研究 较少. 为了阐明共情的遗传和环境作用, 我们首先介 绍双生子研究以探索社会表型的遗传性, 从而评估 遗传与环境对个体的交互作用; 其次，综述环境对共 情的作用, 以及特定的基因及其多态性在个体共情 表型差异中的作用; 最后, 简要介绍共情的影像遗传 学研究, 从而探索具体的心理功能中基因变异对大 脑环路激活的影响.

\section{1 共情的遗传和环境效应}

双生子研究是人们了解遗传和环境对人类个性 和行为表型影响程度的重要研究方法, 根据同卵双 生子 (monozygotic twins) 和异卵双生子 (dizygotic twins)的基因共享程度和表型相似度来确定个性和行 为的遗传基础, 进而探究遗传和环境因素所造成的 相对影响. 同卵双生子几乎共享了 $100 \%$ 的遗传基础, 所以同卵双生子之间的差异主要来源于环境的不同; 异卵双生子共享了 50\%的遗传基础, 他们之间的差 异来自于遗传和环境的共同作用. 在对共情行为表 型的遗传学研究中, Knafo等人 ${ }^{[84]}$ 对七项双生子共情 个体差异的遗传性研究(总共1655名被试数据)进行 荟萃分析, 结果发现遗传因素占了共情差异的 $35 \%$. 这七项研究中, 有六项研究发现, 认知共情和情感共 情的个体差异分别有 30\%和 $26 \%$ 是由遗传因素所导 致. Knafo等人 ${ }^{[85]}$ 早期的研究揭示了遗传与环境之间 的交互作用: 第一, 较低的经济环境或医疗风险会降 低遗传的影响. 换句话说, 只有在优越的环境中儿童 潜在的共情基因才能得到完全的发展. 然而, 在恶劣 的环境中, 环境因素主导了共情能力的发展. 第二, 共情遗传效应随着年龄的增长而愈加明显. 第三, 共 
情能力越高的个体受遗传的影响越大.

很多环境因素可能会影响到共情及其相关行为, 如家庭成员的特质、父母教养方式、人际关系和学校 教育等 ${ }^{[86,87]}$. 研究发现, 父母的特质与儿童的共情相 关行为存在相关性. 例如, 母亲有较高的共情水平 时, 女孩也会表现出较高的共情水平 ${ }^{[88]}$. 母亲的共 情水平与男孩的共情水平不存在关联性, 但是父亲 的共情水平与男孩的共情水平呈正相关关系 ${ }^{[89]}$. 这 可能是儿童更愿意去模仿同性别的家长或更容易受 到同性别家长的引导. 更进一步, Tucker等人 ${ }^{[00]}$ 发现, 10 20岁的女孩的共情能力与比其年长2 3岁姐姐的 共情能力呈显著的正相关关系, 而男孩的共情能力 与年长的哥哥的共情能力也呈类似的关系. 该研究 者认为, 年纪较小的儿童可能会模仿或认同家中年 长的同性别儿童的行为. 另外, 儿童在处于压力或应 激期间, 母亲给予的支持行为可以预测儿童的共情 能力 ${ }^{[91]}$, 即支持行为越多, 儿童的共情能力越强. 因 此, 在儿童处于应激或压力状态时, 母亲给予其足够 的支持有利于他们共情能力的培养. 与兄弟姐妹 ${ }^{[90]}$ 、 父母和同伴 ${ }^{[92,93]}$ 之间亲切融洽的关系也有利于个体 共情能力的发展. 例如, 一项对青少年的研究发现, 相比于与父母和同伴之间为低水平安全关系的个体, 与父母和同伴之间存在较高水平安全关系的个体对 他人表现出更多的共情反应 ${ }^{[92]}$. 长期处于消极和负 面的环境会影响个体感受和表达共情的能力. 例如, Cheng等人 ${ }^{[94]}$ 发现, 与非医疗相关职业的被试相比, 医护人员在观看他人疼痛时的共情反应更弱. 但是 很多医学院校和医疗机构采用相应的教学方式对医 学生或医护人员的共情能力进行训练发现 ${ }^{[95 ~ 98], ~ 人 ~}$ 际技能训练、角色扮演、文学和艺术作品的研读等方 法都可以让医护人员更好地理解他人的情感、痛苦和 遭遇, 从而改善其共情反应.

\section{2 特定基因对共情的影响}

\subsection{1 催产素受体基因}

目前发现, 催产素与不同的神经发育障碍有关, 尤其是社会情感障碍方面, 这些障碍主要表现为社 会情感功能受损, 例如ASD 和精神分裂症 ${ }^{[99]}$. 同时, 催产素的表达和受体密度都会影响个体的社会行为和 认知, 包括个体的社会交往、共情反应和情绪调节等. Rodrigues等人 ${ }^{[100]}$ 研究发现, 催产素受体 (oxytocin receptor)基因rs53576位点上的基因型与被试的行为
共情和特质共情相关, 表现为在 RMET(行为共情)和 IRI问卷(特质共情)测量结果中, 与 $G G$ 型被试相比较, 携带至少一个A-allele的被试 $(A A / A G)$ 表现出更低的 行为和特质共情. Smith等人 ${ }^{[101]}$ 对该基因位点的研究 也得到了类似的结果, 通过测试了51名健康的男性 被试, 他们发现与 $A A / A G$ 型被试相比较, $G G$ 型被试展 现出更高的共情关怀水平和同情反应, 并且在感知 他人痛苦时反应更加强烈. $\mathrm{Wu}$ 等人 ${ }^{[102]}$ 发现, $A A / A G$ 型个体患ASD的可能性更高, 且表现出更低的父母 敏感性(parental sensitivity). Uzefovsky等人 ${ }^{[103]}$ 使用 IRI对 367 名健康成人进行测量发现，催产素受体基 因中rs53576位点上携带A-allele的被试在IRI量表中 情感共情部分(共情关怀, 个人痛苦)得分更低. 此外, 催产素受体基因 rs2254298位点上的基因型与情感共 情也相关. 一项对精神分裂症的研究发现 ${ }^{[99]}$, 携带 至少一个A-allele的精神分裂症患者的情感共情能力 比携带 $G G$ 型基因的患者更高, 主要体现在 $A A / A G$ 型 患者在IRI中的共情关怀(情感共情)维度得分更高. 而 且, $A A / A G$ 型患者在精神病理学阳性阴性量表(分数越 高症状越严重)中的得分比 $G G$ 型个体的更高. 该研究 表明, 催产素受体基因 rs2254298的变异与精神病患 者的共情功能和症状严重程度相关.

\subsection{2 多巴胺受体基因}

共情遗传基础的另一个关注点是多巴胺受体基 因. Poletti等人 ${ }^{[104]}$ 探索了精神分裂症患者的儿茶酚O-甲基转移酶(catechol-O-methyltransferase, COMT) 基因变异对多巴胺能(作用于多巴胺, 泛指与多巴胺 有关的物质或活动, 例如囊泡单胺转运蛋白 2 、多巴 胺转运体和多巴胺受体等)传输(dopaminergic transmission)的影响, 从而研究这种影响是否在患者感知 压力和共情的神经关联性方面存在差异. COMT基因 第3外显子区 $G 1974-A$ 单核苷酸置换导致其蛋白第 158 号缬氨酸 $(\mathrm{Val})$ 被蛋氨酸 $(M e t)$ 替代, 使得酶活性显 著降低. COMT中 $\operatorname{Val}(158)$ 没有被替代则酶活性更高, 而被 $M e t$ 替代则酶活性更低. 因此, COMT 基因 $\operatorname{Val}(158)$ 和Met $(r s 4680)$ 多态性变异会影响酶的活性, 相比于Met/Met携带者, Val/Val携带者的酶活性更高, 较高的酶活性会降低突触后神经元的多巴胺能刺 激 ${ }^{[105]}$. 因此, 与携带Met/Met 的患者相比, 携带 Val/Val 的患者在IRI的个人痛苦分量表中得分更高, 且在共 情相关任务中与共情相关脑区 (如 ACC) 的激活强度 更弱. 该研究认为, 共情反应的强度似乎受到多巴胺 
能信号传递的影响. 此外, 多巴胺受体D4(D4 dopamine receptor, DRD4)中48碱基对序列重复七次的 $D R D 4-7 R$ 基因与父母关怀和子女共情能力存在非常 大的相关性 ${ }^{[106]}$. Knafo 等人 ${ }^{[84]}$ 研究发现, 携带 $D R D 4-7 R$ 基因的儿童的共情关怀能力与母亲的消极 性(maternal negativity)(如恶性言语、身体上的强迫、 很少的爱)存在显著的负相关, 即母亲表现出越多的 消极性, 则孩子的共情关怀能力越低, 但是没有携带 $D R D 4-7 R$ 基因的儿童在母亲消极性和共情关怀之间 没有相关关系.

\subsection{3 五差色胺转运蛋白(5-HTTLPR)基因}

一些学者已经证明了五羟色胺(serotonin，5-HT) 与情绪反应之间存在一定的关联性 ${ }^{[107,108]}$. 因此, 在 对共情的研究中, 研究人员的兴趣点主要在 5-HT转 运蛋白(serotonin transporter, 5-HTTLPR)基因启动子 区的多态性上, 这与 5-HT基因的转录有关 ${ }^{[109]}$. 目前 对5-HTTLPR基因多态性的研究中, Gyurak等人 ${ }^{[110]}$ 发 现其多态性可能与不同的情绪反应相关联. 该研究 比较 5-HTTLPR 基因中分别携带 $S L$ 或 $L L$ 等位基因 的个体发现, 携带两个短的 5-HTTLPR等位基因 (SS5-HTTLPR)的个体在观看他人痛苦的电影之后, 共情 反应更加强烈. 而且, 在观看迤尤处境的录像带时, $S S-5-H T T L P R$ 携带者会表现出更加丰富的面部情绪 表情, 如愤怒和大笑. 因此, 该研究证明, SS-5$H T T L P R$ 携带者可能对他人的情绪和共情反应更加 强烈.

\subsection{4 锌指蛋白(ZNF804A) 基因}

有研究者使用影像遗传学(imaging genetics)的方 法发现了位于锌指蛋白 804A (zinc finger protein 804A, ZNF804A)内含子区内的rs1344706位点的多态 性影响认知共情, 且这种多态性与精神分裂症和双 向人格障碍存在显著的关联 ${ }^{[111,112]}$. 精神分裂症患者 的 ToM 在行为和神经水平上都存在障碍 ${ }^{[113]}$. Walter 等人 ${ }^{[114]}$ 探讨了精神分裂症风险等位基因携带对 ToM 网络激活的影响. 在实验中, 109 名健康被试(其中 18 名被试在 $r s 1344706$ 基因位点的基因型为 $C C$ 型, 49名 为 $C A$ 型, 42 名为 $A A$ 型) 被要求对相继呈现并表达了一 个完整故事的三张卡通图片中人物的情感状态进行 推测 (认知共情)或计算人物的个数(控制条件). 结果 显示, 被试携带的 $A$ 等位基因越多, 在背内侧前额 叶、左后侧STS/TPJ和后扣带皮层(posterior cingulate cortex, PCC) 等脑区 (ToM网络的关键脑区) 的激活越
弱, 即这些脑区的激活程度为 $C C$ 型 $>C A$ 型 $>A A$ 型. 此 外, 这些风险基因携带者的左侧 IFG(涉及镜像神经 元)和左下顶叶 (人类镜像神经元系统的一部分)激活 也很弱 ${ }^{[15]}$, 后来的研究也复制出了类似的结果 ${ }^{[115]}$. 这些结果显示, 精神分裂症风险等位基因与ToM工作 网络激活异常相关. 也就是说, ZNF804A(rs 1344706) 基因的多态性与人类的ToM工作网络的异常相关.

\section{4 共情的遗传-环境-内分泌-大脑理论框架}

综上所述，共情的两种成分具有不同的神经基 础, 且共情能力受到多种因素的调节, 如遗传和环境 等. 结合健康被试和脑损伤患者的研究发现, 情感共 情涉及到的特异性脑区主要有 IFG、脑岛和ACC; 认 知共情的特异性脑区主要是 vmPFC. 对共情的内分 泌研究发现, 催产素可能会促进个体的共情反应, 而 睪丸素则会弱化个体的共情反应, 且产前睪丸素可 能会影响个体共情能力的发育. 此外, 对共情的社会 环境和遗传学研究发现, 家庭成员的特质、父母教养 方式、学校教育和人际关系等环境因素与催产素受 体、DRD4-7R、5-HTTLPR和ZNF804A等基因的多态 性都会影响共情反应和共情能力的发展. 因此, 我们 认为共情特质和共情反应(情绪识别/感染/模仿、眼神 读心和情感/绪理解)可能取决于以下过程, 即个体的 成长环境、遗传因素和内分泌三者相互影响, 且内分 泌与共情相关脑区的活动相互作用; 同时, 遗传和环 境因素会分别作用于大脑; 最后, 遗传、环境、内分 泌和大脑共同作用，从而影响共情及其行为反应. 所 以, 共情受到遗传-环境-内分泌-大脑共同调节(图1).

\section{5 展望}

虽然当前对共情的研究已取得显著的进展, 但 仍存在很多不足, 未来对共情的研究可以从以下几 方面进行:

首先, 通过结合正常人和脑损伤患者的神经影 像结果, 可以揭示参与共情的特异性脑区, 但值得注 意的是, 目前所得到的这些脑损伤患者的神经影像 学结果可能还需要更多的研究去证实其可靠性. 因为 这些研究往往是在患者经历损伤一段时间后才进行 的, 而大脑在损伤之后会有自动修复的功能 ${ }^{[116,117]}$. 因此，基于脑损伤患者的脑成像结果可能无法反映 出已经得到了重组或者恢复的损伤脑区. 针对这一 问题，未来对共情神经机制的研究可以考虑在损伤 


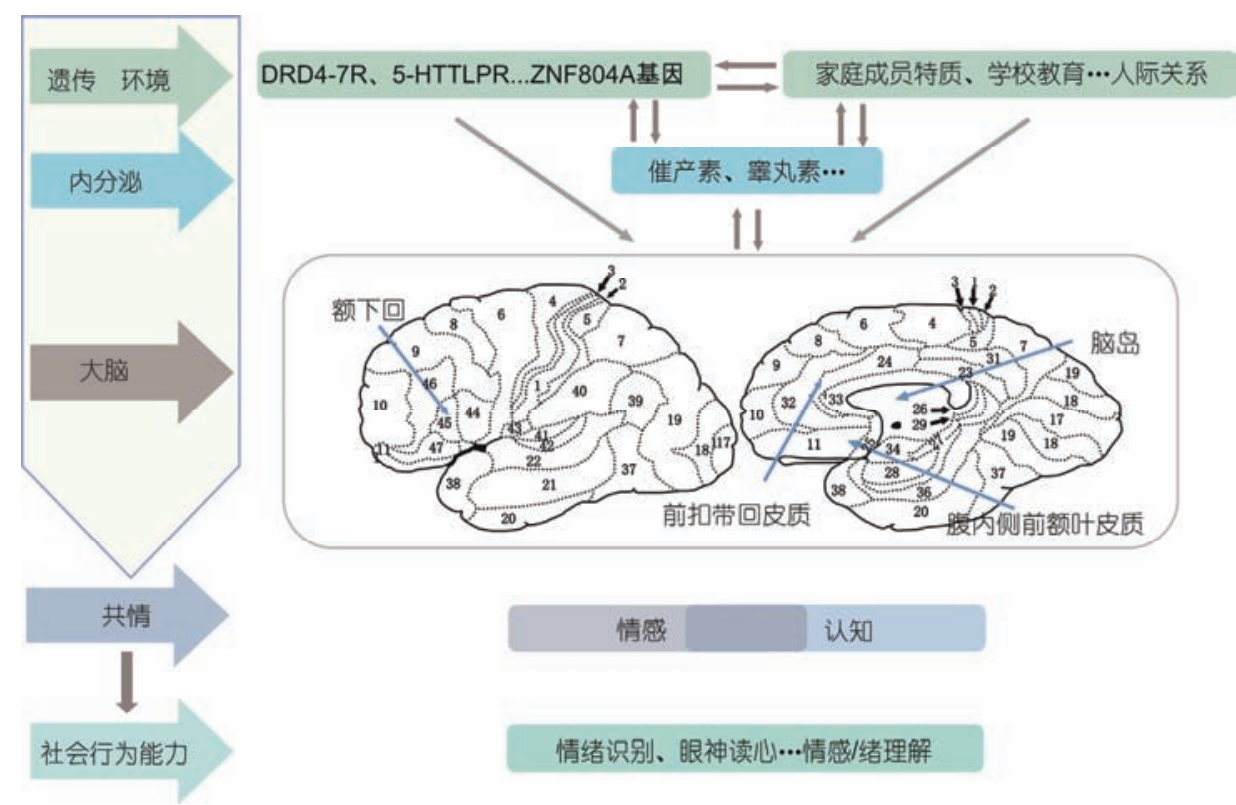

图 1 共情相关的遗传-环境-内分泌-大脑理论框架. 遗传、环境、内分泌和大脑共同作用, 从而影响共情及其社会行为反应能力

Figure 1 The theoretical framework of genetics-environment-endocrine-brain about empathy. Empathy was jointly influenced by genetics, environment, endocrine and brain

发生后较短时间内对其进行研究, 并开展纵向追踪 研究, 以更好地证实共情的特异性脑区. 此外, 近年 来的研究证明, 一些低等动物 (如啮齿类、鸟类和蚁 类)之间也存在共情行为 ${ }^{[10,118]}$. 因此, 未来的研究可 以跨物种进行, 如对低等动物的相关脑区进行干预, 并整合健康人和脑损伤患者的研究, 从进化和发展 的视角去探索共情的神经机制与行为学反应, 进而 为存在共情缺陷的患者提供合理有效的治疗方案. 同时, 当前在激素和基因对共情影响方面的研究仍 值得深人探讨. 例如, 在催产素对特定面部表情进行 识别的研究中, 有一些研究的实验结果仍存在争议, 未来可探索这些研究所得到的争议结果是由于个体 差异还是实验范式或其他的原因所导致的, 进而确 定催产素是如何对共情产生影响的. 而且, 目前仍无 法确定催产素和睪丸素对认知共情和情感共情都存 在影响, 还是只对其中一个成分存在影响. 另外, 可 以结合动物的研究, 通过敲除动物的特定基因, 以检 验相应基因的缺失对激素水平和共情的影响, 从而 探索出特定基因是如何影响共情加工的.

其次, 未来可以从遗传、环境、内分泌和大脑等 因素出发, 全面深人地探讨影响共情的机制. 例如, 在个体的毕生发展中, 激素水平会影响基因的表 达 $^{[119]}$. 然而, 激素水平的变化是否是因为受到了基
因变异的影响? 此外, 基因多态性会导致大脑激活 水平或功能连接上的差异, 这种差异是由于基因多 态性直接影响大脑结构和功能的发展所导致的, 还 是多态性通过影响激素的作用效果而间接导致的? 同时, 大脑结构和功能与激素分泌是如何相互作用 的? 遗传、环境、内分泌和大脑等因素在个体毕生发 展的不同阶段是如何发挥作用的? 现阶段的研究大 多是在被试量较小的基础上孤立其中一种或两种因 素来探讨其与共情的关联性, 无法从根本上解决以 上一系列的问题. 因此, 未来有必要在大样本研究的 基础上开展纵向研究, 综合考量多个因素(遗传、环 境、内分泌和大脑)对共情的影响, 并探究在个体毕 生发展过程中这些因素是如何交互作用而使得共情 发生变化的, 从而揭示共情特质及其行为差异产生 的原因, 进而推进共情领域的理论研究. 例如, 通过 结合使用全基因组测序、脑电(electroencephalogram, EEG)、脑磁(magnetoencephalography, MEG)、磁共振 成像(magnetic resonance imaging, MRI)、血液检测和 问卷等测量技术，合力探究遗传(基因)、大脑(结构和 功能)、内分泌(性激素)、环境(家庭成员的特质、父 母教养方式、学校教育和人际关系)等特征与共情的 关联特性，在进一步拓展人体共情信息处理机制的 相关理论的前提下, 探究(1)共情能力及其行为的个 
体差异及(2)与共情相关的事件(如亲社会行为, 情绪 识别/理解)的产生原因及其机制; 从而提取客观、精 确的与个体共情相关的特异性指标, 为今后的共情 研究建立一个共情数据常模, 提供一个大型的以共 情为基础的数据平台, 进而为培养人们的共情能力、 促进亲社会行为提供可参考的依据, 且为一些情绪 性障碍疾病(ASD、精神分裂症)的诊断及治疗提供一 定的基础指标.

最后, 提高共情研究的生态效度, 促进基础共情 研究成果向社会生活和临床实践转化, 以有效地改 善个体的共情能力及其行为, 从而促进人与人之间 的交往, 进而促进社会和谐与进步, 真正意义上实现 “老吾老, 以及人之老; 幼吾幼, 以及人之幼”. 例如, 在社会生活中, 父母和教育机构可以根据已有的研
究发现，在不同时期采用恰当的方式(如言传身教、支 持鼓励)对孩子进行抚养和教育，从而培养孩子的共 情能力; 消极和负面的环境会阻碍儿童共情能力的 发展, 所以社会和儿童监护人有必要为其创造一个 积极正面的环境以促进儿童共情能力的发展. 临床 实践中, 针对临床环境当中医护人员和患者之间的 共情，采用相应的设备实时记录医患之间共情时的 生理变化, 以深人探讨共情在临床实践中的意义. 医 疗机构应采取合理有效的方式对医护人员或医学生 进行共情教育和培养, 从而改善他们在临床环境中 的共情反应. 基础研究人员应与临床工作者进行密 切合作, 将基础的共情研究推广到临床实践, 探究如 何利用基础研究获得的结果促进医护人员与患者之 间的共情，从而增强临床疗效和改善医患关系.

\section{参考文献}

Bernhardt B C, Singer T. The neural basis of empathy. Annu Rev Neurosci, 2012, 35: 1-23

Decety J, Michalska K J. Neurodevelopmental changes in the circuits underlying empathy and sympathy from childhood to adulthood. Dev Sci, 2010, 13: 886-899

Leigh R, Oishi K, Hsu J, et al. Acute lesions that impair affective empathy. Brain, 2013, 136: 2539-2549

Huang H Q, Su Y J. The development of empathy across the lifespan: A perspective of double processes (in Chinese). Psychol Dev Edu, 2012, 28: 434-441 [黄翌青, 苏彦捷. 共情的毕生发展: 一个双过程的视角. 心理发展与教育, 2012, 28: 434-441]

Dziobek I, Rogers K, Fleck S, et al. Dissociation of cognitive and emotional empathy in adults with Asperger syndrome using the Multifaceted Empathy Test (MET). J Autism Dev Disord, 2008, 38: 464-473

Wai M, Tiliopoulos N. The affective and cognitive empathic nature of the dark triad of personality. Pers Individ Dif, 2012, 52: 794-799

Batson C D, Shaw L L. Evidence for altruism: Toward a pluralism of prosocial motives. Psychol Inq, 1991, 2: 107-122

Eisenberg N, Eggum N D, Giunta L D. Empathy-related responding: Associations with prosocial behavior, aggression, and intergroup relations. Soc Issues Policy Rev, 2010, 4: 143-180

Decety J. Empathy: From bench to bedside. J Cogn Psychother, 2013, 27: 191-192

Chen J. Empathy for distress in humans and rodents. Neurosci Bull, 2017, doi: 10.1007/s 12264-017-0135-0

Singer T, Critchley H D, Preuschoff K, et al. A common role of insula in feelings, empathy and uncertainty. Trends Cogn Sci, 2009, 13: $334-340$

2 Marsh A A, Finger E C, Fowler K A, et al. Empathic responsiveness in amygdala and anterior cingulate cortex in youths with psychopathic traits. J Child Psychol Psychiat, 2013, 54: 900-910

Hurlemann R, Patin A, Onur O A, et al. Oxytocin enhances amygdala-dependent, socially reinforced learning and emotional empathy in humans. J Neurosci, 2010, 30: 4999-5007

Shamay-Tsoory S G, Aharon-Peretz J, Perry D. Two systems for empathy: A double dissociation between emotional and cognitive empathy in inferior frontal gyrus versus ventromedial prefrontal lesions. Brain, 2009, 132: 617-627

Walter H. Social cognitive neuroscience of empathy: Concepts, circuits, and genes. Emot Rev, 2012, 4: 9-17

Shamay-Tsoory S G. The neural bases for empathy. Neuroscientist, 2011, 17: 18-24

Dronkers N F, Wilkins D P, van Valin R D, et al. Lesion analysis of the brain areas involved in language comprehension. Cognition, 2004, 92: 145-177

Dal Monte O, Krueger F, Solomon J M, et al. A voxel-based lesion study on facial emotion recognition after penetrating brain injury. Soc Cogn Affect Neurosci, 2012, 8: 632-639

Hillis A E. Inability to empathize: Brain lesions that disrupt sharing and understanding another's emotions. Brain, 2014, 137: 981-997

Fauci A S. Harrison's Principles of Internal Medicine. Vol. 2. New York: Mcgraw-hill, 1998 
21 Christopoulos G I, Tobler P N, Bossaerts P, et al. Neural correlates of value, risk, and risk aversion contributing to decision making under risk. J Neurosci, 2009, 29: 12574-12583

22 Ross E D, Monnot M. Neurology of affective prosody and its functional-anatomic organization in right hemisphere. Brain Lang, 2008, 104: 51-74

23 Bodini B, Iacoboni M, Lenzi G L. Acute stroke effects on emotions: An interpretation through the mirror system. Curr Opin Neurol, 2004, 17: 55-60

24 Shamay-Tsoory S. The neuropsychology of empathy: Evidence from lesion studies. Revue Neuropsychol, 2015, 7: 237-243

25 Chakrabarti B, Bullmore E, Baron-Cohen S. Empathizing with basic emotions: Common and discrete neural substrates. Soc Neurosci, 2006, 1: 364-384 Jabbi M, Swart M, Keysers C. Empathy for positive and negative emotions in the gustatory cortex. NeuroImage, 2007, 34: 1744-1753 Craig A D, Craig A. How do you feel now? The anterior insula and human awareness. Nat Rev Neurosci, 2009, 10: 59-70 Craig A. Significance of the insula for the evolution of human awareness of feelings from the body. Ann N Y Acad Sci, 2011, 1225: $72-82$

29 Sanfey A G, Rilling J K, Aronson J A, et al. The neural basis of economic decision-making in the ultimatum game. Science, 2003, 300: $1755-1758$

30 Bush G, Luu P, Posner M I. Cognitive and emotional influences in anterior cingulate cortex. Trends Cogn Sci, 2000, 4: 215-222

31 Lamm C, Decety J, Singer T. Meta-analytic evidence for common and distinct neural networks associated with directly experienced pain and empathy for pain. NeuroImage, 2011, 54: 2492-2502

32 Brown S, Gao X, Tisdelle L, et al. Naturalizing aesthetics: Brain areas for aesthetic appraisal across sensory modalities. NeuroImage, 2011, 58: 250-258

33 Mesulam M, Mufson E J. Insula of the old world monkey: Architectonics in the insulo-orbito-temporal component of the paralimbic brain. J Comp Neurol, 1982, 212: 1-22

34 Viskontas I V, Possin K L, Miller B L. Symptoms of frontotemporal dementia provide insights into orbitofrontal cortex function and social behavior. Ann N Y Acad Sci, 2007, 1121: 528-545

35 Hung Y, Smith M L, Taylor M J. Development of ACC-Activations in processing unattended fear. NeuroImage, 2012, 60: 545-552

36 Hadjikhani N, Zürcher N R, Rogier O, et al. Emotional contagion for pain is intact in autism spectrum disorders. Transl Psychiatry, 2014, 4: e 343

37 Kim E J, Sidhu M, Gaus S E, et al. Selective frontoinsular von Economo neuron and fork cell loss in early behavioral variant frontotemporal dementia. Cereb Cortex, 2012, 22: 251-259

38 Butti C, Santos M, Uppal N, et al. Von Economo neurons: Clinical and evolutionary perspectives. Cortex, 2013, 49: 312-326

39 Boucher O, Rouleau I, Lassonde M, et al. Social information processing following resection of the insular cortex. Neuropsychologia, 2015, 71: 1-10

40 Driscoll D M, Dal Monte O, Solomon J, et al. Empathic deficits in combat veterans with traumatic brain injury: A voxel-based lesion-symptom mapping study. Cogn Behav Neurol, 2012, 25: 160-166

41 Baron-Cohen S. Autism: The empathizing-systemizing (E-S) theory. Ann N Y Acad Sci, 2009, 1156: 68-80

42 Mitchell J P, Macrae C N, Banaji M R. Dissociable medial prefrontal contributions to judgments of similar and dissimilar others. Neuron, 2006, 50: 655-663

43 Shamay-Tsoory S G, Aharon-Peretz J. Dissociable prefrontal networks for cognitive and affective theory of mind: A lesion study. Neuropsychologia, 2007, 45: 3054-3067

44 Clark L, Bechara A, Damasio H, et al. Differential effects of insular and ventromedial prefrontal cortex lesions on risky decision-making. Brain, 2008, 131: 1311-1322

45 Urry H L, van Reekum C M, Johnstone T, et al. Amygdala and ventromedial prefrontal cortex are inversely coupled during regulation of negative affect and predict the diurnal pattern of cortisol secretion among older adults. J Neurosci, 2006, 26: 4415-4425

46 Shamay-Tsoory S G, Tomer R, Berger B D, et al. Impaired "affective theory of mind" is associated with right ventromedial prefrontal damage. Cogn Behav Neurol, 2005, 18: 55-67

47 Shamay-Tsoory S G, Shur S, Barcai-Goodman L, et al. Dissociation of cognitive from affective components of theory of mind in schizophrenia. Psychiatry Res, 2007, 149: 11-23

48 Shamay-Tsoory S G, Aharon-Peretz J, Levkovitz Y. The neuroanatomical basis of affective mentalizing in schizophrenia: Comparison of patients with schizophrenia and patients with localized prefrontal lesions. Schizophr Res, 2007, 90: 274-283

49 Hooker C I, Bruce L, Lincoln S H, et al. Theory of mind skills are related to gray matter volume in the ventromedial prefrontal cortex in schizophrenia. Biol Psychiatry, 2011, 70: 1169-1178 
Heinrichs M, von Dawans B, Domes G. Oxytocin, vasopressin, and human social behavior. Front Neuroendocrinol, 2009, 30: 548-557

Carter C S. Neuroendocrine perspectives on social attachment and love. Psychoneuroendocrinology, 1998, 23: 779-818

Insel T R, Young L J. The neurobiology of attachment. Nat Rev Neurosci, 2001, 2: 129-136

Winslow J T, Insel T R. Neuroendocrine basis of social recognition. Curr Opin Neurobiol, 2004, 14: 248-253

Meyer-Lindenberg A, Domes G, Kirsch P, et al. Oxytocin and vasopressin in the human brain: Social neuropeptides for translational medicine. Nat Rev Neurosci, 2011, 12: 524-538

Di Simplicio M, Massey-Chase R, Cowen P J, et al. Oxytocin enhances processing of positive versus negative emotional information in healthy male volunteers. J Psychopharmacol, 2009, 23: 241-248

Marsh A A, Henry H Y, Pine D S, et al. Oxytocin improves specific recognition of positive facial expressions. Psychopharmacology, 2010, 209: 225-232

Schulze L, Lischke A, Greif J, et al. Oxytocin increases recognition of masked emotional faces. Psychoneuroendocrinology, 2011, 36: $1378-1382$

Fischer-Shofty M, Shamay-Tsoory S G, Harari H, et al. The effect of intranasal administration of oxytocin on fear recognition. Neuropsychologia, 2010, 48: 179-184

Adolphs R. Recognizing emotion from facial expressions: Psychological and neurological mechanisms. Behav Cogn Neurosci Rev, 2002, 1: $21-62$

Andari E, Duhamel J R, Zalla T, et al. Promoting social behavior with oxytocin in high-functioning autism spectrum disorders. Proc Natl Acad Sci USA, 2010, 107: 4389-4394

Gamer M, Zurowski B, Büchel C. Different amygdala subregions mediate valence-related and attentional effects of oxytocin in humans. Proc Natl Acad Sci USA, 2010, 107: 9400-9405

Domes G, Lischke A, Berger C, et al. Effects of intranasal oxytocin on emotional face processing in women. Psychoneuroendocrinology, 2010, 35: 83-93

Lischke A, Berger C, Prehn K, et al. Intranasal oxytocin enhances emotion recognition from dynamic facial expressions and leaves eye-gaze unaffected. Psychoneuroendocrinology, 2012, 37: 475-481

De Dreu C K, Kret M E. Oxytocin conditions intergroup relations through upregulated in-group empathy, cooperation, conformity, and defense. Biol Psychiatry, 2016, 79: 165-173

Kret M E, De Dreu C K. Oxytocin-motivated ally selection is moderated by fetal testosterone exposure and empathic concern. Front Neurosci, 2013, 7: 1-9

De Wilde T R W, Ten Velden F S, De Dreu C K W. The neuropeptide oxytocin enhances information sharing and group decision making quality. Sci Rep, 2017, 7: 40622

Guastella A J, Mitchell P B, Dadds M R. Oxytocin increases gaze to the eye region of human faces. Biol Psychiatry, 2008, 63: 3-5

Bartz J A, Hollander E. Oxytocin and experimental therapeutics in autism spectrum disorders. Prog Brain Res, 2008, 170: 451-462

Baron-Cohen S, Wheelwright S, Hill J, et al. The "Reading the Mind in the Eyes" test revised version: A study with normal adults, and adults with Asperger syndrome or high-functioning autism. J Child Psychol Psychiatry, 2001, 42: 241-251

Domes G, Heinrichs M, Michel A, et al. Oxytocin improves "mind-reading” in humans. Biol Psychiatry, 2007, 61: 731-733

Bassil N, Alkaade S, Morley J E. The benefits and risks of testosterone replacement therapy: A review. Ther Clin Risk Manag, 2009, 5: $427-448$

2 Lutchmaya S, Baron-Cohen S. Human sex differences in social and non-social looking preferences, at 12 months of age. Infant Behav Dev, 2002, 25: 319-325

Hoffman M L. Sex differences in empathy and related behaviors. Psychol Bull, 1977, 84: 712-722

Chapman E, Baron-Cohen S, Auyeung B, et al. Fetal testosterone and empathy: Evidence from the empathy quotient (EQ) and the "reading the mind in the eyes" test. Soc Neurosci, 2006, 1: 135-148

Auyeung B, Baron-Cohen S, Chapman E, et al. Foetal testosterone and the child systemizing quotient. Eur J Endocrinol, 2006, 155: S123-S130

6 Baron-Cohen S, Richler J, Bisarya D, et al. The systemizing quotient: An investigation of adults with Asperger syndrome or highfunctioning autism, and normal sex differences. Philos Trans R Soc Lond B Biol Sci, 2003, 358: 361-374

Baron-Cohen S. The extreme male brain theory of autism. Trends Cogn Sci, 2002, 6: 248-254

Palomba S, Marotta R, Di Cello A, et al. Pervasive developmental disorders in children of hyperandrogenic women with polycystic ovary syndrome: A longitudinal case-control study. Clin Endocrinol, 2012, 77: 898-904

Baron-Cohen S, Wheelwright S, Spong A, et al. Studies of theory of mind: Are intuitive physics and intuitive psychology independent. The Core Deficit in Autism and Disorders of Relating and Communicating, 2001, 5: 1-47 
80 van Honk J, Schutter D J, Bos P A, et al. Testosterone administration impairs cognitive empathy in women depending on second-tofourth digit ratio. Proc Natl Acad Sci USA, 2011, 108: 3448-3452

81 Hermans E J, Putman P, Van Honk J. Testosterone administration reduces empathetic behavior: A facial mimicry study. Psychoneuroendocrinology, 2006, 31: 859-866

82 Bos P A, Hofman D, Hermans E J, et al. Testosterone reduces functional connectivity during the 'Reading the Mind in the Eyes' Test. Psychoneuroendocrinology, 2016, 68: 194-201

83 Hermans E J, Ramsey N F, van Honk J. Exogenous testosterone enhances responsiveness to social threat in the neural circuitry of social aggression in humans. Biol Psychiatry, 2008, 63: 263-270

84 Knafo A, Uzefovsky F. Variation in empathy: The interplay of genetic and environmental factors. In: Legerstee M, Haley D W, Bornstein M H, eds. The Infant Mind: Origins of the Social Brain. New York, NY: The Guilford Press, 2013. 97-122

85 Knafo A, Zahn-Waxler C, Davidov M, et al. Empathy in early childhood. Ann N Y Acad Sci, 2009, 1167: 103-114

86 Hastings P D, Utendale W T, Sullivan C. The socialization of prosocial development. In: Grusec J E, Hastings P D, eds. Handbook of Socialization: Theory and Research. New York, NY: The Guilford Press, 2007. 638-664

87 Eisenberg N, Fabes R A, Spinrad T. Handbook of Child Psychology. New Jersey: John Wiley \& Sons, 2006

88 Eisenberg N, Fabes R A, Carlo G, et al. The relations of maternal practices and characteristics to children's vicarious emotional responsiveness. Child Dev, 1992, 63: 583-602

89 Eisenberg N, Fabes R A, Schaller M, et al. The relations of parental characteristics and practices to children's vicarious emotional responding. Child Dev, 1991, 62: 1393-1408

90 Tucker C J, Updegraff K A, McHale S M, et al. Older siblings as socializers of younger siblings' empathy. J Early Adolesc, 1999, 19: 176-198

91 Bryant B K. Mental health, temperament, family, and friends: Perspectives on children's empathy and social perspective taking. In: Eisenberg N, Strayer J, eds. Empathy and Its Development. Cambridge: Cambridge University Press, 1987. 245-270

92 Laible D J, Carlo G, Raffaelli M. The differential relations of parent and peer attachment to adolescent adjustment. J Youth Adolesc, 2000, 29: 45-59

93 Laible D J, Carlo G, Roesch S C. Pathways to self-esteem in late adolescence: The role of parent and peer attachment, empathy, and social behaviours. J Adolesc, 2004, 27: 703-716

94 Cheng Y, Lin C P, Liu H L, et al. Expertise modulates the perception of pain in others. Curr Biol, 2007, 17: 1708-1713

95 Werner A, Schneider J M. Teaching medical students interactional skills: A research-based course in the doctor-patient relationship. N Engl J Med, 1974, 290: 1232-1237

96 Feighny K M, Arnold L, Monaco M, et al. In pursuit of empathy and its relationship to physician communication skills: Multidimensional empathy training for medical students. Ann Behav Sci Med Educ, 1998, 5: 13-21

97 Starcevic V, Piontek C M. Emphathic understanding revisited: Conceptualization, controversies, and limitations. Am J Psychotherapy, 1997, 51: 317-328

98 Lancaster T, Hart R, Gardner S. Literature and medicine: Evaluating a special study module using the nominal group technique. Med Educ, 2002, 36: 1071-1076

99 Montag C, Brockmann E M, Lehmann A, et al. Association between oxytocin receptor gene polymorphisms and self-rated 'empathic concern' in schizophrenia. PLoS One, 2012, 7: e51882

100 Rodrigues S M, Saslow L R, Garcia N, et al. Oxytocin receptor genetic variation relates to empathy and stress reactivity in humans. Proc Natl Acad Sci USA, 2009, 106: 21437-21441

101 Smith K E, Porges E C, Norman G J, et al. Oxytocin receptor gene variation predicts empathic concern and autonomic arousal while perceiving harm to others. Soc Neurosci, 2014, 9: 1-9

102 Wu S, Jia M, Ruan Y, et al. Positive association of the oxytocin receptor gene (OXTR) with autism in the Chinese Han population. Biol Psychiatry, 2005, 58: 74-77

103 Uzefovsky F, Shalev I, Israel S, et al. Oxytocin receptor and vasopressin receptor 1a genes are respectively associated with emotional and cognitive empathy. Horm Behav, 2015, 67: 60-65

104 Poletti S, Radaelli D, Cavallaro R, et al. Catechol-O-methyltransferase (COMT) genotype biases neural correlates of empathy and perceived personal distress in schizophrenia. Comprehen Psychiatry, 2013, 54: 181-186

105 Egan M F, Goldberg T E, Kolachana B S, et al. Effect of COMT Val108/158 Met genotype on frontal lobe function and risk for schizophrenia. Proc Natl Acad Sci USA, 2001, 98: 6917-6922

106 Belsky J, Pluess M. Beyond diathesis stress: Differential susceptibility to environmental influences. Psychol Bull, 2009, 135: 885-908

107 Beevers C G, Wells T T, Ellis A J, et al. Association of the serotonin transporter gene promoter region (5-HTTLPR) polymorphism with biased attention for emotional stimuli. J Abnorm Psychol, 2009, 118: 670-681 
108 Hysek C M, Schmid Y, Simmler L D, et al. MDMA enhances emotional empathy and prosocial behavior. Soc Cogn Affect Neurosci, 2013, 9: 1645-1652

109 Heils A, Teufel A, Petri S, et al. Allelic variation of human serotonin transporter gene expression. J Neurochem, 1996, 66: 2621-2624

110 Gyurak A, Haase C M, Sze J, et al. The effect of the serotonin transporter polymorphism (5-HTTLPR) on empathic and self-conscious emotional reactivity. Emotion, 2013, 13: 25-35

111 O'donovan M C, Craddock N, Norton N, et al. Identification of loci associated with schizophrenia by genome-wide association and follow-up. Nat Genet, 2008, 40: 1053-1055

112 Williams H J, Craddock N, Russo G, et al. Most genome-wide significant susceptibility loci for schizophrenia and bipolar disorder reported to date cross-traditional diagnostic boundaries. Human Mol Genet, 2010, 20: 387-391

113 Bora E, Veznedaroğlu B, Vahip S. Theory of mind and executive functions in schizophrenia and bipolar disorder: A cross-diagnostic latent class analysis for identification of neuropsychological subtypes. Schizophr Res, 2016, 17: 500-505

114 Walter H, Schnell K, Erk S, et al. Effects of a genome-wide supported psychosis risk variant on neural activation during a theory-ofmind task. Mol Psychiatry, 2011, 16: 462-470

115 Mohnke S, Erk S, Schnell K, et al. Further evidence for the impact of a genome-wide-supported psychosis risk variant in ZNF804A on the Theory of Mind Network. Neuropsychopharmacology, 2014, 39: 1196-1205

116 Luria A R, Naydin V L, Tsvetkova L S, et al. Restoration of higher cortical function following local brain damage. Behav Brain Sci, 1969, 52: 687-718

117 Guggenmos D J, Azin M, Barbay S, et al. Restoration of function after brain damage using a neural prosthesis. Proc Natl Acad Sci USA, 2013, 110: 21177-21182

118 Chen J, Li Z, Lü Y F, et al. Empathy for pain: A novel bio-psychosocial-behavioral laboratory animal model (in Chinese). Acta Physiol Sin, 2015, 67: 561-570 [陈军, 李震, 吕云飞, 等. 疼痛共情: 一个新的生物-社会心理-行为学实验动物模型. 生理学报, 2015, 67: 561-570]

119 McCarthy M M, Arnold A P. Reframing sexual differentiation of the brain. Nat Neurosci, 2011, 14: 677-683 
Summary for “共情：遗传-环境-内分泌-大脑机制”

\title{
Empathy: The genetics-environment-endocrine-brain mechanism
}

\author{
YANG Ye $e^{1,2,3}$, TANG Yi ${ }^{1}$, PENG WeiWei ${ }^{4}$, LU XueJing ${ }^{2,3^{*}}$, HÜ Li $^{1,2, *}$ \& CHEN Jun ${ }^{5}$ \\ ${ }^{1}$ Key Laboratory of Cognition and Personality (Ministry of Education) and School of Psychology, Southwest University, Chongqing 400715, China; \\ ${ }^{2}$ CAS Key Laboratory of Mental Health, Institute of Psychology, Beijing 100101, China; \\ ${ }^{3}$ Department of Psychology, University of Chinese Academy of Sciences, Beijing 100049, China; \\ ${ }^{4}$ Brain Function and Psychological Science Research Center, Shenzhen University, Shenzhen 518060, China; \\ ${ }^{5}$ Institute for Biomedical Sciences of Pain, Tangdu Hospital, The Fourth Military Medical University, Xi'an 710038, China \\ *Corresponding authors, E-mail: luxj@psych.ac.cn; huli@psych.ac.cn
}

As a vital ability for everyday communication and survival in a socical environment, empathy, including affective empathy and cognitive empathy, plays a crucial role in helping individuals feel, understand and share the emotional state of others. Affective empathy was defined as sharing experiences of emotional states perceived in others, including emotional contagion and affective perspective taking; cognitive empathy involves the ability to engage in the cognitive processes of adopting another's psychological point of view as well as understanding other's affective and cognitive mental states, similar to Theory of Mind (ToM). Given that empathy provides the foundation of motivation and emotion in individual moral development, it can be used in clinical practice to optimize the medical treatment by promoting patient-physician communication, reducing patients' anxiety and distress. Thus, it is full of social significance and clinical meaning to comprehensively and deeply explore the biological basis of empathy.

To get a better understanding of empathy, this paper will review relevant research on empathy and list the possible influential factors that may affect one's empathy. First, based on the evidence from neuroimaging study on both healthy individuals and patients with brain lesions, we summarized the neural basis of affective empathy and cognitive empathy. These findings indicate that affective empathy involves specific brain areas, such as insula, anterior cingulate cortex (ACC), and inferior frontal gyrus (IFG). In contrast, ventromedial prefrontal cortex (vmPFC) is specific to cognitive empathy. Second, we expatiated the factors related to endocrine system that may influence individuals' empathy and their related behaviors. Whereas oxytocin facilitates the response of empathy, testerone weakens it. In addition, fetal testosterone would influence the development of individual empathy. Third, the development of empathy can also be affected by the genetic polymorphism of oxytocin receptor (OXTR), zinc finger protein 804A (ZNF804A), serotonin transporter (5-HTTLPR), and D4 dopamine receptor (DRD4). With the development of a child, the influences of environmental factors, such as characteristics of family members, parenting styles, and schooling, on empathy cannot be ignored. Taken all these possible factors into account, a theoretical framework for understanding empathy is proposed, which comprehensively reveals the biological basis of empathy and how it can be affected by genetics, environment, endocrine, and brain. Furthermore, we discussed the shortcomings of previous studies on empathy, mainly due to the small sample size and/or the isolation of influential factors from the whole theoretical framework. Finally, we emphasized that further investigations on empathy should focus on longitudinal studies with large sample size, and make efforts to reveal the interactions of the influential factors (e.g. genetics, environment, endocrine and brain). More importantly, experimental findings of empathy research should be well explained and translated to the social and clinical applications, as the improvement of empathy can be used in promoting social harmony and enhancing clinical treatment.

empathy, affective empathy, cognitive empathy, brain, environment, gene

doi: 10.1360/N972017-00832 